\title{
Radial profile of energetic particles bombarding the substrate in a glow discharge
}

\author{
Juan Carlos Moreno-Marín and Isabel Abril \\ Departament de Física Aplicada, Universitat d'Alacant, Apartat 99, E-03080 Alacant, Spain \\ Rafael Garcia-Molina ${ }^{\text {a) }}$ \\ Departamento de Física, Universidad de Murcia, Apartado 4021, E-30080 Murcia, Spain
}

(Received 21 August 1998; accepted 20 November 1998)

\begin{abstract}
We have analyzed in an interrelated way the main processes that occur in a glow discharge system: generation of the energetic particles that strike the cathode, emission of sputtered and reflected atoms from the cathode, and transport of these particles through the plasma, until their eventual arrival to the substrate. As an output of our calculations we have obtained the radial profiles of the energy and the number of particles that reach the substrate, as a function of the geometrical characteristics and pressure of the discharge. Our results show that the main part of the energy deposited in the substrate comes from the reflected gas atoms, in spite of the larger number of sputtered cathode atoms. (C) 1999 American Vacuum Society. [S0734-2101(99)02702-5]
\end{abstract}

\section{INTRODUCTION}

Glow discharges are extensively studied as they play an important role in plasma processing, such as surface treatment and thin film deposition. During the last decade, there has been an increasing interest in cold plasmas produced by electrical discharges (dc or rf) and their applications, ${ }^{1}$ and much attention has been paid to analyze (experimentally, theoretically and by simulation) glow discharge plasmas of the type used for electronic materials processing. ${ }^{2,3}$ In order to improve the use of cold plasmas in industry, it is necessary to understand the basic mechanisms occurring in these systems and to obtain an optimization of the parameters relevant for the desired applications. The structure and the physical properties (like adhesion, electric conductivity, stoichiometry and strain) of the thin films grown in a glow discharge system strongly depend on the spatial distribution of the energy deposited by the particles that arrives at the substrate. ${ }^{4-6}$

The aim of this article is to calculate the energy distribution and the number of particles that bombard the substrate in a glow discharge sputtering system, as a function of the radial distance from the cathode-substrate axis. Special emphasis will be paid to analyze the role played by those gas particles that, after being reflected in the cathode, reach the substrate with high energy, as compared to the sputtered cathode particles. The effects of the discharge pressure, $p$, and the cathode-substrate distance, $d$, on the radial profile of energy deposited in the substrate, will be discussed in detail for both the sputtered and the reflected fluxes of particles.

We will study a planar dc glow discharge, with the cathode made of molybdenum, using argon as discharge gas, and the substrate being located at the anode; the main geometrical parameters of the system are shown in Fig. 1. In glow discharge sputtering systems, we can distinguish two regions: the dark space of the cathode, where almost all the

${ }^{a)}$ Electronic mail: rgm@fcu.um.es potential fall takes place, and a region that is approximately equipotential, called the plasma region. When ions from the plasma enter in the cathode dark space they are accelerated towards the cathode by the electric field; this flux of ions undergoes charge-exchange collisions with the thermal gas atoms, originating a flux of fast neutral atoms. Both fluxes of energetic particles, ions and neutrals, strike the cathode and produce the emission of sputtered particles and secondary electrons, these latter helping to sustain the discharge. Moreover, a fraction of the incident gas atoms are reflected from the cathode and gives a flux of backscattered gas particles. The fluxes of the sputtered cathode atoms and the reflected gas atoms abandon the cathode and cross the plasma losing energy until they eventually arrive at the substrate.

This article is organized as follows. In the next section, we will present a model for the glow discharge that connects all the above mentioned processes. Then, in Sec. III, we will analyze the dependence of the final results on the parameters that characterize the discharge. The main conclusions of this work will be summarized in Sec. IV.

\section{GLOW DISCHARGE MODEL}

We consider three stages in a glow discharge sputtering system: (a) the acceleration of the gas ions and the generation of the energetic neutral gas particles in the cathode dark space, giving rise to a flux of energetic particles that bombard the cathode; (b) the sputtering of the cathode particles and the reflection of the gas particles at the cathode; and (c) the transport and thermalization through the plasma of the particles emitted from the cathode, until they reach the substrate.

Within the cathode dark space the generation of energetic fluxes that bombard the cathode, which corresponds to the first stage, is dominated by inelastic processes, such as ionization or charge exchange. However, elastic events prevail both in the sputtering and backscattering processes that take place during the second stage, and also are responsible for 


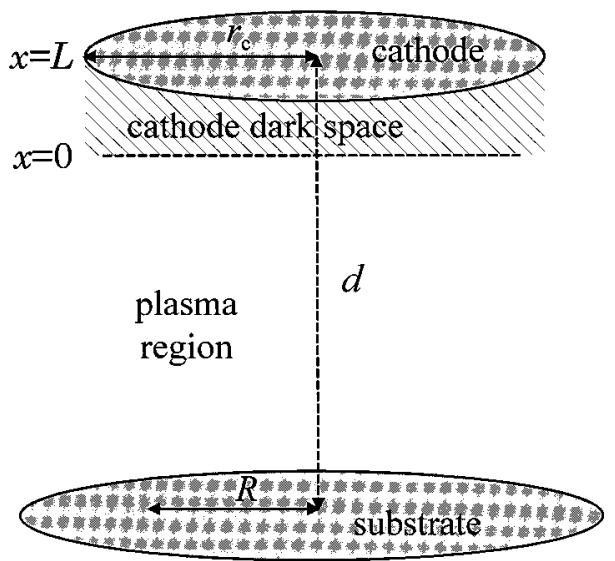

FIG. 1. Schematic view of a glow discharge sputtering system. The gas in the discharge is argon, and the cathode is made of molybdenum. $r_{c}$ is the cathode radius, $d$ is the separation between the cathode and the substrate, $R$ is the radial distance from the cathode-substrate axis, and $L$ is the thickness of the cathode dark space.

the energy degradation of the particles whereas, during the third stage, they travel through the plasma until the substrate.

In order to provide an overall description of the glow discharge sputtering system, we have connected the above mentioned three stages using the results obtained in one stage as input for the next one. The energy distributions of the flux of particles (ions and energetic neutrals) striking the cathode are the input quantities used to calculate the energy and angular distribution of particles emitted (due to sputtering and backscattering) from the cathode, which in its turn constitute the input needed to simulate the transport of these particles through the plasma.

\section{A. Generation of the particles that bombard the cathode}

The first stage in the discharge, i.e., the generation of the energetic fluxes of gas particles in the cathode dark space, is modelled self-consistently using a set of Boltzmann transport equations, together with Poisson's equation. ${ }^{7}$ Three different fluxes of energetic particles are considered: (i) gas ions injected from the plasma or generated by electron ionization collisions, $\quad e\left(E_{0}\right)+\operatorname{Ar}^{0}(k T) \rightarrow e\left(E_{0}-E_{\text {ioniz }}\right)+e(k T)$ $+\operatorname{Ar}^{+}(k T)$; (ii) electrons emitted from the cathode due to the bombardment of energetic particles or due to ionization collisions; and (iii) energetic neutral gas particles resulting from charge-exchange collisions, $\operatorname{Ar}^{+}\left(E_{0}\right)$ $+\operatorname{Ar}^{0}(k T) \rightarrow \operatorname{Ar}^{0}\left(E_{0}\right)+\operatorname{Ar}^{+}(k T)$. $e$ stands for electron, $k T$ is the thermal energy, $E_{0}$ is a generic energy, and $E_{\text {ioniz }}$ is the ionization energy of the gas ions (15 eV for argon).

We define $\Phi_{j}\left(x, E_{0}\right) d E_{0}$ as the number of particles of the $j$ type that are at a distance $x$ from the plasma-dark space interface and that have an energy in the interval $\left(E_{0}, E_{0}\right.$ $\left.+d E_{0}\right)$; these quantities will be normalized at one ion that crosses this interface (which is at $x=0$ ). The index $j$ may be $i$ for ions, $n$ for energetic gas neutrals and $e$ for electrons.
The energy distribution of the ions, $\Phi_{i}\left(x, E_{0}\right)$, is given by the following transport equation:

$$
\begin{aligned}
& \frac{\partial \Phi_{i}\left(x, E_{0}\right)}{\partial x} \\
& =-e \xi(x) \frac{\partial \Phi_{i}\left(x, E_{0}\right)}{\partial E_{0}}+\delta\left(E_{0}\right)\left[\int \frac{\Phi_{i}\left(x, E^{\prime}\right) d E^{\prime}}{\lambda_{\mathrm{cx}}\left(E^{\prime}\right)}\right. \\
& \left.\quad+\int \frac{\Phi_{e}\left(x, E^{\prime}\right) d E^{\prime}}{\lambda_{\text {ioniz }}\left(E^{\prime}\right)}\right]-\frac{\Phi_{i}\left(x, E_{0}\right)}{\lambda_{\mathrm{cx}}\left(E_{0}\right)},
\end{aligned}
$$

where $\xi$ is the electric field. The energy distribution corresponding to the energetic neutral gas atoms, $\Phi_{n}\left(x, E_{0}\right)$, is related with that of the ions through:

$$
\frac{\partial \Phi_{n}\left(x, E_{0}\right)}{\partial x}=\frac{\Phi_{i}\left(x, E_{0}\right)}{\lambda_{\mathrm{cx}}\left(E_{0}\right)} .
$$

And the energy distribution of the electrons in the dark space can be obtained from:

$$
\begin{aligned}
- & \frac{\partial \Phi_{e}\left(x, E_{0}\right)}{\partial x} \\
= & e \xi(x) \frac{\partial \Phi_{e}\left(x, E_{0}\right)}{\partial E_{0}}+\delta\left(E_{0}\right) \int \frac{\Phi_{e}\left(x, E^{\prime}\right) d E^{\prime}}{\lambda_{\text {ioniz }}\left(E^{\prime}\right)} \\
& +\frac{\Phi_{e}\left(x, E_{0}+E_{\text {ioniz }}\right)}{\lambda_{\text {ioniz }}\left(E_{0}+E_{\text {ionz }}\right)}-\frac{\Phi_{e}\left(x, E_{0}\right)}{\lambda_{\text {ioniz }}\left(E_{0}\right)} .
\end{aligned}
$$

In all the above expressions, $e$ is the elementary charge, $\lambda_{\mathrm{cx}}$ and $\lambda_{\text {ioniz }}$ are the mean free path for charge-exchange and ionization processes, respectively, and $\delta(\ldots)$ is Dirac's delta function. The distance $x$ is taken as positive from the plasma-dark space interface towards the cathode (Fig. 1), therefore the ions and energetic neutrals travel towards the cathode and the electrons in the opposite direction.

The spatial distribution of the electric field $\xi(x)$ is calculated self-consistently as a solution of Poisson's equation:

$$
\begin{aligned}
\frac{\partial \xi(x)}{\partial x}= & \frac{1}{\epsilon_{0}}\left[J_{i}(x) \int \frac{\Phi_{i}\left(x, E^{\prime}\right) d E^{\prime}}{\left(2 E^{\prime} / m_{g}\right)^{1 / 2}}\right. \\
& \left.-J_{e}(x) \int \frac{\Phi_{e}\left(x, E^{\prime}\right) d E^{\prime}}{\left(2 E^{\prime} / m_{e}\right)^{1 / 2}}\right],
\end{aligned}
$$

where $m_{g}$ and $m_{e}$ stand for the masses of the gas particles and the electron, respectively, and $\epsilon_{0}$ is the permittivity of vacuum. The total current density is $J=J_{i}+J_{e}$, where $J_{i}$ and $J_{e}$ represent, respectively, the current densities of ions and electrons in the dark space. The boundary conditions satisfied by the energy distributions are $\Phi_{i}\left(x=0, E_{0}\right)=\delta\left(E_{0}\right)$ and $\Phi_{e}\left(x=L, E_{0}\right)=\delta\left(E_{0}\right)$, i.e., the ions are injected from the plasma $(x=0)$ with zero energy and the secondary electrons emitted from the cathode $(x=L)$ also have zero energy.

To do the calculations for a representative situation, we have used the following set of discharge parameters: cathode potential $V_{c}=1000 \mathrm{~V}$, current density $J=10 \mathrm{~A} / \mathrm{m}^{2}$ and gas pressure ranging from 10 to 200 mTorr. The energy distributions of ions and energetic neutrals at the cathode, $\Phi_{i}\left(L, E_{0}\right)$ and $\Phi_{n}\left(L, E_{0}\right)$, respectively, are shown in Fig. 2 for several 


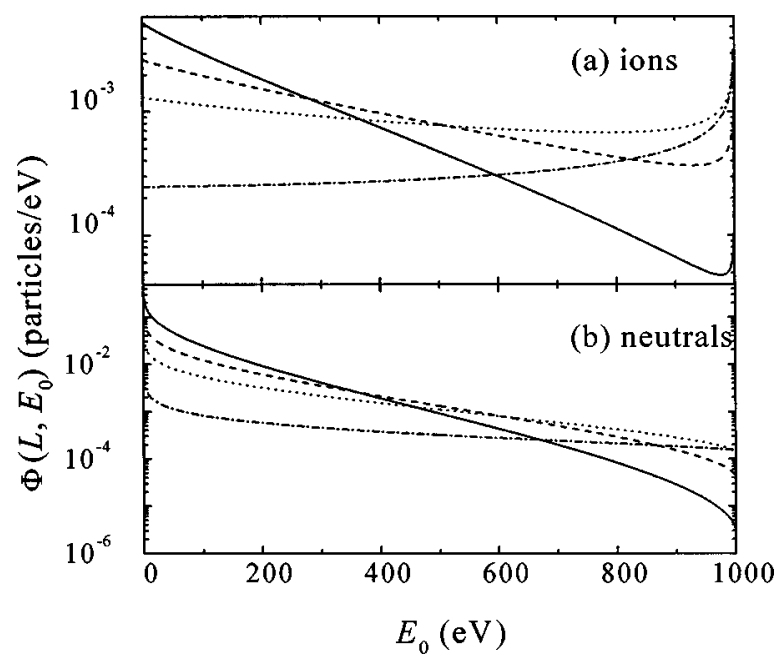

FIG. 2. Energy distribution of the gas particles that bombard the cathode, at $x=L$ : (a) ions, and (b) neutrals. The discharge parameters are $V_{c}=1000 \mathrm{~V}$ and $J=10 \mathrm{~A} / \mathrm{m}^{2}$, and the following gas pressures have been considered: $p$ $=200 \mathrm{mTorr}$ (solid line), $p=100 \mathrm{mTorr}$ (dashed line), $p=50 \mathrm{mTorr}$ (dotted line), and $p=10$ mTorr (dash-dotted line).

values of the gas pressure. The flux of ions obtain energy from the electric field and lose energy by charge-exchange collisions with thermal gas atoms when they travel towards the cathode. As we observe in Fig. 2(a), the energy distribution of ions decreases monotonically with increasing energy because charge-exchange collisions are very efficient processes; only those ions that travel without collisions acquire the maximum energy, $E_{\max } \sim e V_{c}$. When the gas pressure rises there is also an increase in the number of collisions that takes place in the cathode dark space, therefore there is a reduction in the number of ions that do not undergo any collision, and the ion energy distribution shifts towards low energies.

We observe in Fig. 2(b) that the energy distribution of the energetic neutral gas particles at the cathode decreases as the energy increases, because the flux of neutrals obtain its energy directly from charge-exchange collisions with energetic gas ions. It is important to remark the significance of including charge-exchange processes, which generates a cascade of fast neutrals atoms that bombard the cathode. ${ }^{8-10}$ When the gas pressure increases, the behavior of the energy distribution corresponding to the fast neutrals is similar to that of the ions.

The total number of particles (ions and neutrals) that bombards the cathode, per one ion that crosses the plasmadark space interface, is given by

$$
\begin{aligned}
N^{\mathrm{tot}}(L) & =N_{i}^{\mathrm{tot}}(L)+N_{n}^{\mathrm{tot}}(L) \\
& =\int_{0}^{E_{\max }}\left[\Phi_{i}\left(E_{0}, L\right)+\Phi_{n}\left(E_{0}, L\right)\right] d E_{0} .
\end{aligned}
$$

Figure 3(a) shows that the total number of energetic neutrals that arrive at the cathode, $N_{n}^{\text {tot }}(L)$, grows linearly as a function of the discharge pressure, whereas the total number of ions, $N_{i}^{\text {tot }}(L)$, remains close to one. For $p \simeq 20$ mTorr, the

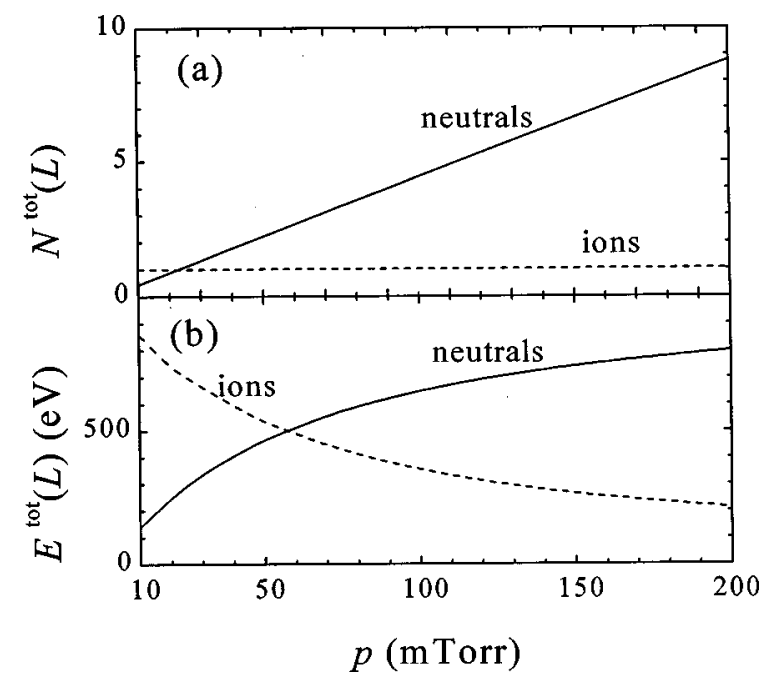

FIG. 3. (a) Total number of energetic neutral gas atoms (solid line) and ions (dashed line) that bombard the cathode, represented as a function of the discharge pressure. (b) Total energy that arrives at the cathode carried by the energetic neutrals (solid line) and the ions (dashed line), as a function of the discharge pressure.

number of ions and neutrals are approximately equal, whereas for $p \simeq 200$ mTorr, there are nine times more energetic neutrals than ions bombarding the cathode.

The total amount of energy deposited at the cathode by the ions and energetic neutrals is given by

$$
\begin{aligned}
E^{\mathrm{tot}}(L) & =E_{i}^{\mathrm{tot}}(L)+E_{n}^{\mathrm{tot}}(L) \\
& =\int_{0}^{E_{\max }}\left[\Phi_{i}\left(E_{0}, L\right)+\Phi_{n}\left(E_{0}, L\right)\right] E_{0} d E_{0} .
\end{aligned}
$$

Figure 3(b) shows $E_{i}^{\text {tot }}(L)$ and $E_{n}^{\text {tot }}(L)$ as a function of the gas pressure. Due to the increase in the number of chargeexchange collisions with the thermal gas atoms, the energy transported by the ions to the cathode decreases monotonically as the pressure increases. The same argument applies to the increase in the amount of energy transported by the fast neutrals as the pressure increases. For $p \simeq 50$ mTorr, the energy transported by ions and neutrals are rather similar, while for $p \simeq 200 \mathrm{mTorr}$, the fast neutrals transport four times more energy than the ions. Therefore it is essential to consider the generation of the flux of fast energetic neutrals in order to describe appropriately the operation of a glow discharge sputtering system.

\section{B. Emission of particles from the cathode}

Next we will study the second stage that takes place in the operation of a glow discharge system, that is, the sputtering of cathode atoms due to the bombardment of energetic gas particles and the backscattering of these gas particles at the cathode.

When an energetic ion bombards a solid, it is neutralized and begins to lose energy into the target. In the range of energies carried by the particles that hit the cathode in a typical glow discharge (see Fig. 2), the energy loss is mainly due to atomic collisions with the target nuclei, giving rise to 
a collision cascade inside the solid; consequently some of the cathode atoms obtain enough energy to leave the cathode. This phenomenon is known as sputtering. Besides the sputtering process, another effect of the collision cascade is the reflection of the energetic gas particles after they bombard the cathode.

The Monte Carlo code TOPS $^{11}$ allows one to calculate the energy and angular distribution of the sputtered and reflected particles at the cathode, produced when a single projectile strikes the cathode with a given energy. This code ${ }^{11}$ is based on the binary collision approximation to evaluate the collision cascade induced in the cathode; the $\mathrm{Kr}-\mathrm{C}$ screened $\mathrm{Cou}-$ lomb potential ${ }^{12}$ is used to describe in a realistic way the atomic mixing (primary recoil and cascade events) that takes place in the solid, and the energy losses due to inelastic events are also considered in the simulation. ${ }^{13}$

Using the energy distribution for the flux of energetic gas particles (ions and neutrals) that bombards the cathode with energy $E_{0}, \Phi_{i}\left(L, E_{0}\right)+\Phi_{n}\left(L, E_{0}\right)$, which was calculated in Sec. II A, we can write down the energy distribution of the sputtered (or reflected) particles emitted at the cathode, $\Psi_{s(r)}(E)$, as follows

$\Psi_{s(r)}(E)=\int_{0}^{E_{\max }}\left[\Phi_{i}\left(L, E_{0}\right)+\Phi_{n}\left(L, E_{0}\right)\right] \psi_{s(r)}\left(E_{0}, E\right) d E_{0}$,

where $\psi_{s(r)}\left(E_{0}, E\right)$ is the energy distribution of sputtered (or reflected) particles produced by a single incident projectile with energy $E_{0}$.

Due to charge-exchange collisions in the dark space, there is a reduction of the energy carried by the gas ions when they strike the cathode. Consequently, as the sputtering yield $Y$ is roughly proportional to the energy of the incident particles, ${ }^{14}$ a decrease in the number of sputtered particles would be expected. However, this trend is compensated by the additional sputtering produced by the flux of energetic neutral gas atoms originated in the charge-exchange events. ${ }^{15}$

The energy distribution of the reflected gas particles, $\Psi_{r}(E)$, is shown in Fig. 4 for different discharge gas pressures. As the pressure increases the energy distribution concentrates at lower energies; this behavior is correlated with the reduction of energy carried by the particles that bombard the cathode (see Fig. 2). The second peak in these distributions (clearly observable at $E \sim 10 \mathrm{eV}$ for $p=200$ mTorr, but appearing as an almost flat hill at $E \sim 160 \mathrm{eV}$ for $p=50$ mTorr), is due to atoms that have been reflected after a single collision inside the cathode. The inset of Fig. 4 shows a comparison between the energy distribution of sputtered atoms, $\Psi_{s}$, and reflected gas particles, $\Psi_{r}$, for a gas pressure $p=200$ mTorr. The shape of $\Psi_{s}$, peaked at low energies $(\sim 5-10 \mathrm{eV})$ remains almost independent of the discharge gas pressure. ${ }^{15}$ However, $\Psi_{r}$ has a significant contribution at high energies. In Table I, we present for different gas pressures the sputtering yield, $Y$, divided by the reflection coefficient, $R$, that is,

$$
\frac{Y}{R}=\frac{\int \Psi_{s}(E) d E}{\int \Psi_{r}(E) d E} .
$$

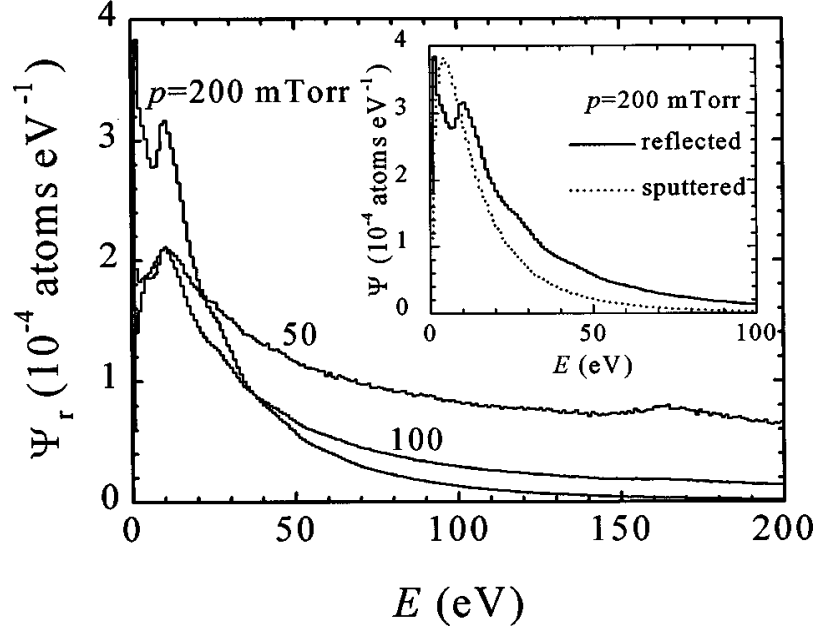

FIG. 4. Energy distribution of reflected argon atoms at the cathode. The parameters of the discharge are $V_{c}=1000 \mathrm{~V}$ and $J=10 \mathrm{~A} / \mathrm{m}^{2}$; several working gas pressures have been considered: $p=200$ mTorr (solid line), $p$ $=100 \mathrm{mTorr}$ (dashed line) and $p=50 \mathrm{mTorr}$ (dotted line). The inset compares, for $p=200 \mathrm{mTorr}$, the energy distributions at the cathode of the sputtered molybdenum atoms, $\Psi_{s}$ (dotted line), and the reflected argon atoms, $\Psi_{r}$ (solid line)

This ratio compares the number of particles that are emitted from the cathode as sputtered or reflected ones. For the system analyzed in this work, where there is a large mass difference between the gas (Ar) and the cathode (Mo) atoms, it is found that the ratio $Y / R$ decreases as the gas pressure increases. A discussion of $Y$ and $R$ for Ar bombarding different targets at various energies is provided in Ref. 16. Table I also displays the ratio between the total energy carried by the sputtered and reflected particles emitted at the cathode:

$$
\frac{E_{s}^{\mathrm{emitted}}}{E_{r}^{\mathrm{emitted}}}=\frac{\int \Psi_{s}(E) E d E}{\int \Psi_{r}(E) E d E} .
$$

From the data shown in Table I, it is clear that although there are more sputtered particles than reflected ones, the latter are emitted with higher energies, which can also be seen in Fig. 4. Therefore the flux of reflected particles will be responsible for the greater part of the energy that arrives at the substrate. . $^{4,17}$

TABLE I. Ratios between the sputtering yield and the reflection coefficient, $Y / R$, and between the energy carried by the sputtered and the reflected particles, $E_{s}^{\mathrm{emitted}} / E_{r}^{\mathrm{emitted}}$ at the cathode, calculated for several discharge gas pressures.

\begin{tabular}{ccc}
\hline \hline$p$ (mTorr) & $Y / R$ & $E_{s}^{\text {emitted }} / E_{r}^{\text {emitted }}$ \\
\hline 10 & 3.993 & 0.786 \\
30 & 3.826 & 0.781 \\
50 & 3.475 & 0.766 \\
100 & 1.986 & 0.674 \\
200 & 0.687 & 0.394 \\
\hline \hline
\end{tabular}




\section{Transport of particles through the plasma}

The next stage in our study is the transport through the plasma of the energetic particles emitted from the cathode. We will use the three-dimensional Monte Carlo code TRAP ${ }^{18}$ to analyze the motion and thermalization of these fluxes of particles. The main advantages of the code TRAP are the following: it allows the use of any energetic and angular distributions for the particles emitted at the cathode, it provides a realistic treatment of the collisions in the plasma, and, finally, the geometry of the discharge chamber as well as the position and size of the cathode and the substrate can be easily taken into account. ${ }^{18}$

Since the concentration of the plasma neutral gas atoms is higher than that of the energetic particles, we have considered that when the latter travel through the plasma they lose energy only by collisions with the plasma neutral gas atoms; therefore in what follows we neglect interactions between particles of the moving fluxes as well as electronic energy loss. The transport through the discharge chamber of each particle emitted from the cathode is simulated by the TRAP code as follows: first, the position, energy and direction when the particle leaves the cathode are chosen, and second, its trajectory is followed calculating the scattering angle and the energy loss each time a collision takes place; the transport process comes to an end when the particle reaches the substrate or when it is thermalized. This sequence constitutes a history, which is repeated until a good statistics is obtained. We have taken $0.04 \mathrm{eV}$ for the thermalization energy, which corresponds to a discharge temperature of $\sim 300 \mathrm{~K}$.

The departure point of the emitted particles from the cathode is taken randomly from a homogeneous distribution, therefore the spatial distribution of the particles is a function of the cathode geometry. In this work, the cathode is a circle with radius $r_{c}=5 \mathrm{~cm}$. The initial energy and angular distributions of the emitted particles are taken according to the results obtained using the Monte Carlo code TOPS, discussed in Sec. II B, as well as to the sputtering yield $Y$ and the reflection coefficient $R$.

The interatomic potential used to describe the scattering processes in the plasma is a basic parameter because it determines the cross section, the energy loss and the scattering angle at each collision. The TRAP code ${ }^{18}$ simulates atomic binary collisions using a screened Coulomb potential; it describes the elastic collisions between the plasma gas atoms and the energetic particles emitted from the cathode by a power-law interatomic potential $V(r) \propto \lambda_{m} r^{-1 / m}$, where $r$ is the distance between the interacting particles. According to the masses and relative energy of the interacting particles, we have chosen ${ }^{18}$ the potential parameter $m=1 / 6$ and the collisional cross section parameter $\lambda_{m}=6,{ }^{19}$ which provide a satisfactory agreement with experimental data. ${ }^{20}$

In this work we want to analyze the spatial profile of the energy deposited into the substrate due to the fluxes of both the sputtered and reflected particles from the cathode. The dependence with the pressure and the geometrical characteristics of the discharge chamber is discussed in Ref. 15 for the sputtered flux of particles.

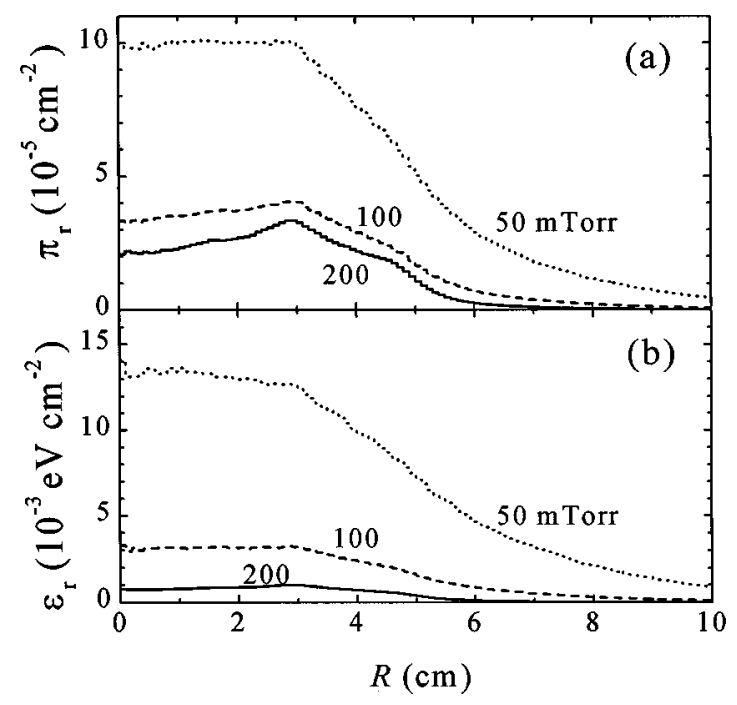

FIG. 5. (a) Radial profile, per unit area, of the number of argon atoms that, after being reflected from the cathode, arrive at the substrate. (b) Radial profile, per unit area, of the energy carried by these atoms when they reach the substrate. The discharge voltage and current density are, respectively, $V_{c}=1000 \mathrm{~V}$ and $J=10 \mathrm{~A} / \mathrm{m}^{2}$; the geometrical parameters of the discharge are $r_{c}=5 \mathrm{~cm}$ and $d=5 \mathrm{~cm}$; several gas pressures have been considered: $p$ $=200 \mathrm{mTorr}$ (solid line), $p=100 \mathrm{mTorr}$ (dashed line) and $p=50 \mathrm{mTorr}$ (dotted line).

In what follows, we will analyze the spatial distribution of the energy deposited into the substrate by the flux of gas particles reflected from the cathode. In order to quantify the substrate irradiation as a function of the radial distance $R$ from its center (which is concentric with that of the cathode, see Fig. 1), we have calculated the radial profiles of the energy $\varepsilon_{r}(R)$ and the number of particles $\pi_{r}(R)$, both per unit area, that reach the substrate.

Figure 5 illustrates the influence of the discharge pressure on $\varepsilon_{r}(R)$ and $\pi_{r}(R)$. The cathode-substrate distance is $d$ $=5 \mathrm{~cm}$. As it can be seen, a greater substrate irradiation, both in energy and in number of particles, takes place when the pressure decreases, because the energetic particles undergo fewer collisions during their travel through the plasma. However, the substrate region that is irradiated homogeneously, represented by the plateau in each curve, grows as the pressure increases, although at the expense of receiving a lower energy and number of particles. It is also interesting to know the total energy deposited in the substrate by the reflected flux of gas particles [given by $2 \pi \int \varepsilon_{r}(R) R d R$ ] and the total number of particles [given by $2 \pi \int \pi_{r}(R) R d R$ ], as a function of the gas pressure. From the results presented in Fig. 5, we can conclude that the number of particles that arrive at the substrate decreases by a factor of 3 when the discharge pressure changes from 50 to 100 mTorr, but this reduction is only a factor 1.5 when the pressure goes from 100 to 200 mTorr. The total energy that arrives at the substrate decreases by a factor of $\sim 5$ when the pressure increases from 50 to 100 mTorr and also from 100 to 200 mTorr. The average energy per each particle arriving at the substrate [i.e., $\int \varepsilon_{r}(R) R d R / \int \pi_{r}(R) R d R$ ] is $144 \mathrm{eV}$ at 50 mTorr, $93 \mathrm{eV}$ at $100 \mathrm{mTorr}$ and $33 \mathrm{eV}$ at $200 \mathrm{mTorr}$. These 
results are a consequence of the dependence of initial energy distribution of the reflected particles as a function of the discharge pressure (Fig. 4), and they show how effective is the stopping by the plasma as the pressure increases.

In conclusion, we have seen that as the discharge pressure increases, the energy transported by the gas particles reflected from the substrate present a more homogeneous radial profile, although the average energy per particle diminishes.

\section{RESULTS AND DISCUSSION}

In this section, we will analyze the spatial profile of the energy deposited in the substrate, comparing the effect due to the flux of reflected gas particles at the cathode with that due to the flux of sputtered cathode particles. The main differences in the energy deposited by these fluxes of particles stem from their different initial energy distribution at the cathode (see the inset in Fig. 4) and their transport through the plasma.

The sputtered particles are ejected from the cathode with an energy distribution that is peaked at low energy (5-10 $\mathrm{eV}$ ), while the gas particles reflected at the cathode present a high energy tail in their energy distribution (as has been shown in Fig. 4) depending on the discharge pressure. Concerning the transport stage, the ratio between both energytransfer collision cross sections involved in the interactions we are considering is $\sigma_{\mathrm{Ar}-\mathrm{Ar}} / \sigma_{\mathrm{Mo}-\mathrm{Ar}} \simeq 0.7$. This result indicates that, during their travel through the plasma, the atoms reflected at the cathode lose less energy than the sputtered ones, ${ }^{17}$ therefore the former will play a much more significant role in the deposition of energy at the substrate than the latter.

The radial profile of the deposited energy $\varepsilon(R)$ and the number of particles $\pi(R)$, both per area, that arrives at the substrate are shown in Fig. 6, for a discharge pressure of 100 mTorr and for several values of the cathode-substrate distance $d$. We have separated the contribution to $\varepsilon(R)$ and $\pi(R)$ due to the flux of reflected gas particles from that due to flux of sputtered particles. The former appears as the shaded region, whereas the latter is the white area between each pair of curves. The profile due to both, reflected and sputtered particles, is given in each case by the upper curve.

The main remark derived from Fig. 6 is the different role played by the sputtered and the reflected particles concerning the energy and the number of particles that reach the substrate. Comparing the shaded versus the white areas in the left part of Fig. 6, we can see that the reflected particles carry the main part of the energy that arrives at the substrate and also are the responsible of the shape of the radial profile of the energy deposited in the substrate. The number of sputtered atoms that land into the substrate (right part of Fig. 6) is appreciably larger than the number of reflected particles, but the former contribute little to the energy deposition.

The substrate area with an (almost) homogeneous energy deposition, represented by the plateau in the curves at the left of Fig. 6, increases the farther the cathode-substrate distance $d$ is, but there is a reduction of the total energy deposited in

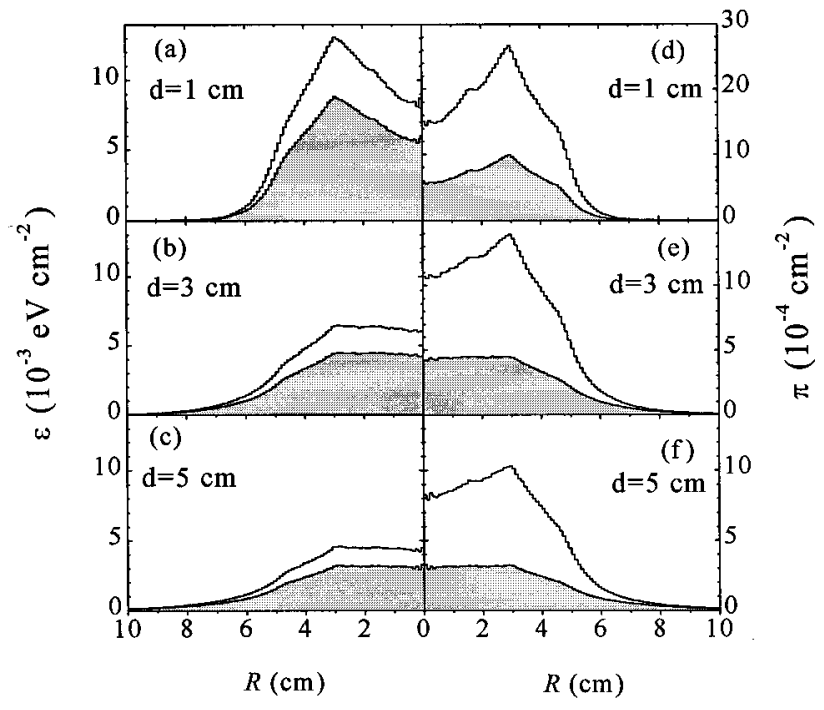

FIG. 6. Radial profiles, per unit area, of the energy (left part) and the number of particles (right part) incident onto the substrate, for several cathodesubstrate distances $d$. The contribution to these profiles from the argon atoms reflected is represented by the shadowed area, while the contribution due to the molybdenum atoms sputtered at the cathode corresponds to the white area comprised between both curves in each figure. The highest curve in each figure is the total radial profile, due to sputtered atoms plus reflected particles at the substrate. The gas pressure, discharge voltage and current density are, respectively, $p=100 \mathrm{mTorr}, V_{c}=1000 \mathrm{~V}$ and $J=10 \mathrm{~A} / \mathrm{m}^{2}$. The radius of the cathode is $r_{c}=5 \mathrm{~cm}$ and the cathode-substrate distance $d$ is indicated in each figure.

the substrate. However, the radial profile of the total number of particles that arrive at the substrate has a shape that is rather independent of $d$. In any case, a better spatial homogeneity is observed in the incidence of energy than in the number of particles that arrive at the substrate. At the present we are not aware of experimental data corresponding to the system discussed in this paper, therefore a comparison with our results has not been possible.

The results presented in this work are a consequence of two important facts. First, the initial energetic distributions of particles reflected and sputtered from the cathode show that practically all the high-energy emitted particles are reflected gas particles; whereas the low-energy emitted particles are either predominately reflected or sputtered, depending on gas pressure. Second, transport through the plasma is clearly advantageous to the high-energy reflected gas particles. A significant number of sputtered particles will be thermalized in their travel to the substrate and, therefore, the main part of the energy deposited onto the substrate will be provided by the flux of reflected gas particles.

\section{CONCLUDING REMARKS}

We have discussed the generation and transport of energetic particles through a planar dc glow discharge, paying special attention to clarify the importance of the two kinds of particles (sputtered cathode atoms and reflected plasma atoms) in the energy deposition at the substrate.

Three different, but correlated, processes have been considered. In the cathode dark-space region we have used a set 
of Boltzmann transport equations, together with Poisson's equation, to calculate the energy distribution of the gas particles (ions and fast neutrals) that bombard the cathode, as well as the electric field in the sheath. ${ }^{7}$ The energy and angular distributions of both the reflected and the sputtered particles emitted from the cathode were calculated using the Monte Carlo code TOPS. ${ }^{11}$ And finally, the transport and thermalization of these fluxes of particles through the plasma were evaluated with the Monte Carlo code TRAP. ${ }^{18}$ The results calculated at each stage were used as input in the next stage, in such a manner that all the processes in the discharge system are interrelated.

As a final output of our calculations, we have obtained the radial profiles of the energy and the number of particles that bombard the substrate, for each type of energetic particles, reflected gas particles and sputtered particles from the cathode. This has clarified the role played by each one of these types of particles in the energy deposition at the substrate.

For the characteristics of the glow discharge, we have discussed in this work, our results show that although the cathode sputtered particles represent the greater flux of particles, the high-energy reflected gas particles are mainly responsible for the energy deposition on the substrate. Also, our calculations have shown how the spatial homogeneity in the energy deposited onto the substrate can be modified by properly varying the characteristic parameters of the plasma discharge.

\section{ACKNOWLEDGMENTS}

This work is supported by the Spanish Dirección General de Investigación Científica y Técnica (Projects PB95-0689 and PB96-1118). The authors thank J. J. Jiménez-Rodríguez (Universidad Complutense, Madrid) for providing us the computer code TOPS.
${ }^{1}$ B. Chapman, Glow Discharge Processes. Sputtering and Plasma Etching (Wiley, New York, 1980).

${ }^{2}$ A. J. van Roosmalen, J. A. G. Baggerman, and S. J. H. Brader, Dry Etching for VLSI (Plenum, New York, 1991).

${ }^{3}$ M. A. Lieberman and A. J. Lichtenberg, Principles of Plasma Discharges and Materials Processing (Wiley, New York, 1994).

${ }^{4}$ H. F. Winters, H. J. Coufal, and W. Eckstein, J. Vac. Sci. Technol. A 11, 657 (1993).

${ }^{5}$ E. Kay, F. Parmigiani, and W. Parrish, J. Vac. Sci. Technol. A 5, 44 (1987).

${ }^{6}$ S. M. Rossnagel, Thin Film Processes II. Glow Discharge Plasmas and Sources for Etching and Deposition (Academic, Orlando, 1991).

${ }^{7}$ I. Abril, Comput. Phys. Commun. 51, 413 (1988); I. Abril, A. Gras-Martí, and J. A. Vallés-Abarca, J. Vac. Sci. Technol. A 4, 1773 (1983); Vacuum 37, 391 (1987).

${ }^{8}$ I. Abril, A. Gras-Martí, and J. A. Vallés-Abarca, Phys. Rev. A 28, 3677 (1983); J. Phys. D 17, 1841 (1984).

${ }^{9}$ Z. Wronski, J. L. Sullivan, and S. O. Saied, J. Phys. D 25, 1607 (1992).

${ }^{10}$ A. Bogaerts, M. van Straaten, and R. Gijbels, Spectrochim. Acta B 50, 179 (1995).

${ }^{11}$ A. M. C. Pérez-Martín, and J. J. Jiménez-Rodríguez, Vacuum 39, 701 (1989); 42, 537 (1991).

${ }^{12}$ W. D. Wilson, L. G. Haggmark, and J. P. Biersack, Phys. Rev. B 15, 2458 (1977).

${ }^{13}$ J. Lindhard and M. Scharff, Phys. Rev. 124, 128 (1961).

${ }^{14} \mathrm{P}$. Sigmund, in Topics in Applied Physics, edited by R. Behrisch (Springer, Berlin, 1981), Chap. II, Vol. 47.

${ }^{15}$ J. C. Moreno-Marín, I. Abril, R. Garcia-Molina, A. M. C. Pérez-Martín, and J. J. Jiménez-Rodríguez, Vacuum 48, 685 (1997).

${ }^{16}$ I. Abril, A. Gras-Martí, J. J. Jiménez-Rodríguez, V. Konoplev, J. C. Moreno-Marín, and A. M. C. Pérez-Martín, Trends in Vacuum Sci. Tech. 1, 251 (1993).

${ }^{17}$ J. C. Moreno-Marín, I. Abril, A. Gras-Martí, V. Konoplev, J. J. JiménezRodríguez, and A. M. C. Pérez-Martín, Vacuum 45, 1135 (1994).

${ }^{18}$ A. Gras-Martí, J. A. Vallés-Abarca, and J. C. Moreno-Marín, in PlasmaSurface Interactions and Processing of Materials, edited by O. Auciello, A. Gras-Martí, J. A. Vallés-Abarca, and D. L. Flamm (Kluwer, Boston, 1990), p. 135.

${ }^{19}$ J. Lindhard, V. Nielsen, and M. Scharff, Mat. Fys. Medd. Dan. Vid. Selsk. 36, 10 (1968).

${ }^{20}$ R. S. Robinson, J. Vac. Sci. Technol. 16, 185 (1979). 